\title{
Orientation diagnostique devant les anomalies du temps de saignement, du temps de céphaline activé, du temps de Quick et de l'international normalized ratio
}

\section{Item $N^{\circ} 172$}

Le TS (temps de saignement), le TCA (temps de céphaline activé), le TQ (temps de Quick) et l'INR (international normalized ratio) sont indiqués :

(1) soit pour le dépistage des anomalies congénitales ou acquises de l'hémostase;

(2) soit pour l'évaluation d'un risque hémorragique connu et/ou la surveillance d'un traitement anti-thrombotique.

Dans le cadre du dépistage des anomalies de l'hémostase, le recours à ces examens n'est en aucun cas systématique. En effet, en l'absence d'éléments évocateurs, la découverte fortuite d'une anomalie de l'hémostase est rare et souvent de faible valeur prédictive positive quant à ses manifestations opératoires potentielles. Le dépistage d'anomalies de l'hémostase repose d'abord sur une recherche préalable d'argument(s)

médecine buccale chirurgie buccale

VOL. $9, N^{\circ} 3$ 2003

page 185 pertinent(s) en faveur d'un trouble de l'hémostase, par un interrogatoire (tableau 1) et par l'observation d'éventuels signes cliniques évocateurs. Ce sont les réponses aux questions et l'examen clinique qui orienteront l'indication et le choix des éventuels examens demandés. 
Tableau 1: Questionnaire de dépistage d'un risque hémorragique. Une histoire clinique positive, ou un interrogatoire impossible, pose l'indication d'un bilan biologique simplifié de l'hémostase (TS, TCA, TQ et numération plaquettaire). D'après : Indications des examens préopératoires, ANDEM, 1992.

médecine buccale chirurgie buccale

VOL. $9, \mathrm{~N}^{\circ} 3$ 2003

page 186

\begin{tabular}{|c|c|}
\hline Type A & $\begin{array}{l}\text { - Le patient a-t-il saigné plus de } 24 \text { heures ou a-t-il nécessité une transfusion sanguine à la } \\
\text { suite d'un acte chirurgical tel que circoncision, amygdalectomie, appendicectomie ou suture } \\
\text { de peau ? } \\
\text { - Est-ce qu'après une extraction dentaire s'est produite une gingivorragie prolongée toute } \\
\text { la nuit ou une récidive hémorragique après } 24 \text { heures ayant nécessité une nouvelle consul- } \\
\text { tation pour un traitement dentaire ou médical? } \\
\text { - Existe-t-il des antécédents d'hématurie inexpliquée? } \\
\text { - Le patient a-t-il consommé au cours des deux semaines précédentes des médicaments } \\
\text { contenant des salicylés ou des anti-inflammatoires non stéroïdiens? } \\
\text { - L'examen clinique met-il en évidence des ecchymoses anormales, des pétéchies, des } \\
\text { signes de malnutrition ou de malabsorption, des signes de maladie hépatique ou hémato- } \\
\text { logique? }\end{array}$ \\
\hline Type B & $\begin{array}{l}\text { - Les incidents précédents se sont-ils produits chez des parents ou des hommes du côté } \\
\text { maternel ? } \\
\text { - Existe-t-il des ecchymoses faciles sans cause apparente? } \\
\text { - Est-ce qu'une épistaxis a nécessité un tamponnement chirurgical pour assurer l'hémo- } \\
\text { stase? } \\
\text { - Est-ce que les sites de ponction veineuse saignent plus de } 15 \text { minutes après l'application } \\
\text { du pansement? } \\
\text { - A-t-on déjà signalé au patient une tendance anormale au saignement? }\end{array}$ \\
\hline
\end{tabular}

Une réponse de type $A$ = deux réponses de type $B$ = histoire clinique positive.

\section{TEMPS DE SAIGNEMENT (TS)}

Le TS est un test global d'exploration de l'hémostase primaire (Fig. 1). C'est le seul test réalisé in vivo. Il dépend de :

(1) la qualité et la quantité des plaquettes,

(2) la thrombogénicité de la paroi vasculaire,

(3) deux facteurs plasmatiques (facteur Von

Willebrand et fibrinogène).

Le TS est défini par le temps séparant la section d'un groupe de petits capillaires et l'arrêt du saignement ainsi provoqué. Il peut être évalué par deux méthodes :

\section{La méthode de DUKE}

Il s'agit d'une incision pratiquée au lobe de l'oreille à l'aide d'un vaccinostyle. La valeur normale est inférieure ou égale à 4 minutes. Cette méthode est abandonnée du fait de son manque de sensibilité et de reproductibilité.

\section{Da méthode IVY}

Une brèche vasculaire est pratiquée sur la face antérocubitale de l'avant bras, à l'aide d'un dispositif standardisé automatique. Elle est réalisée sous pression constante de $40 \mathrm{mmHg}$ obtenue en gonflant un brassard à tension. Le sang est recueilli toutes les 30 secondes sur un buvard Whatman $n^{\circ} 1$ sans toucher la plaie. Le résultat est le temps où l'on observe la dernière goutte de sang ayant traversé le buvard.

II en existe 2 variantes:

\section{- IVY 3 points}

La brèche vasculaire consiste en 3 piqûres réalisées au moyen de microlances. La valeur normale est inférieure à 5 minutes.

- IVY incision

La brèche vasculaire consiste en 2 incisions parallèles ou perpendiculaires au pli du coude réalisées au moyen de lames. Elle est considérée 


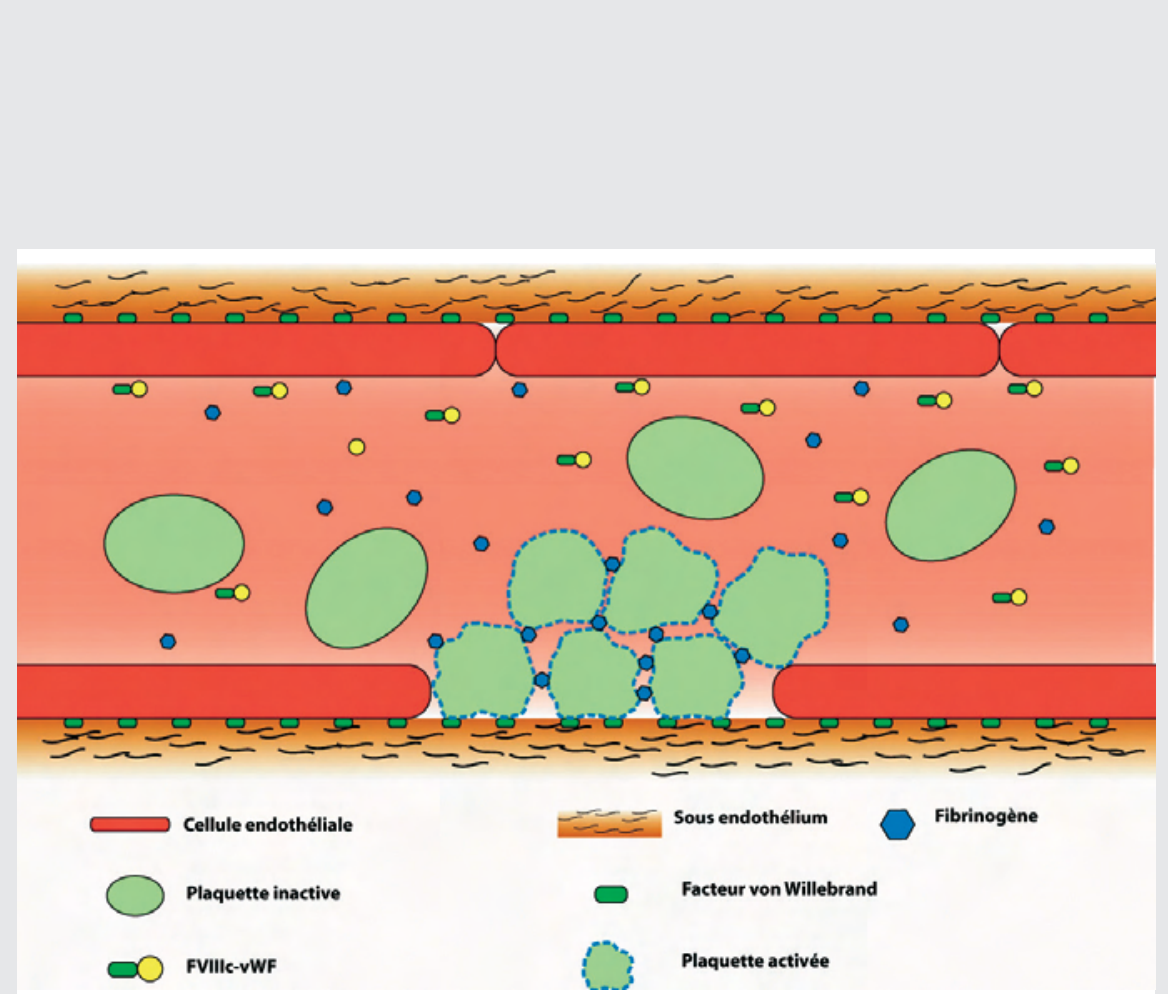

Figure 1 : Schéma de l'hémostase primaire : interactions entre les différents acteurs de la formation du thrombus blanc.

comme la technique de référence. La valeur normale est inférieure à 8 minutes. L'allongement du TS est considéré comme significatif au delà de 10 minutes.

Le TS n'est plus actuellement un examen prescrit à titre systématique lors d'un bilan d'hémostase préopératoire. II est inutile chez les malades thrombopéniques ou traités par un inhibiteur des fonctions plaquettaires. En revanche, il reste un examen utile pour le diagnostic des thrombopathies et de certaines formes de la maladie de Willebrand.

\section{TEMPS DE CÉPHALINE ACTIVÉE (TCA)}

Le TCA est un test global d'exploration de la coagulation sanguine (Fig 2). II explore, in vitro :

- la voie endogène (ou voie de la phase contact),

- le tronc commun (complexe de la prothrombinase, thrombinoformation, fibrinoformation).

Ce test consiste à mesurer le temps de coagulation à $37^{\circ} \mathrm{C}$ d'un plasma pauvre en plaquettes, anticoagulé par recueil sur citrate de sodium $(0,113 \mathrm{M})$, après avoir :

- activé la voie de la phase de contact par du kaolin, silice ou de l'acide ellagique ;

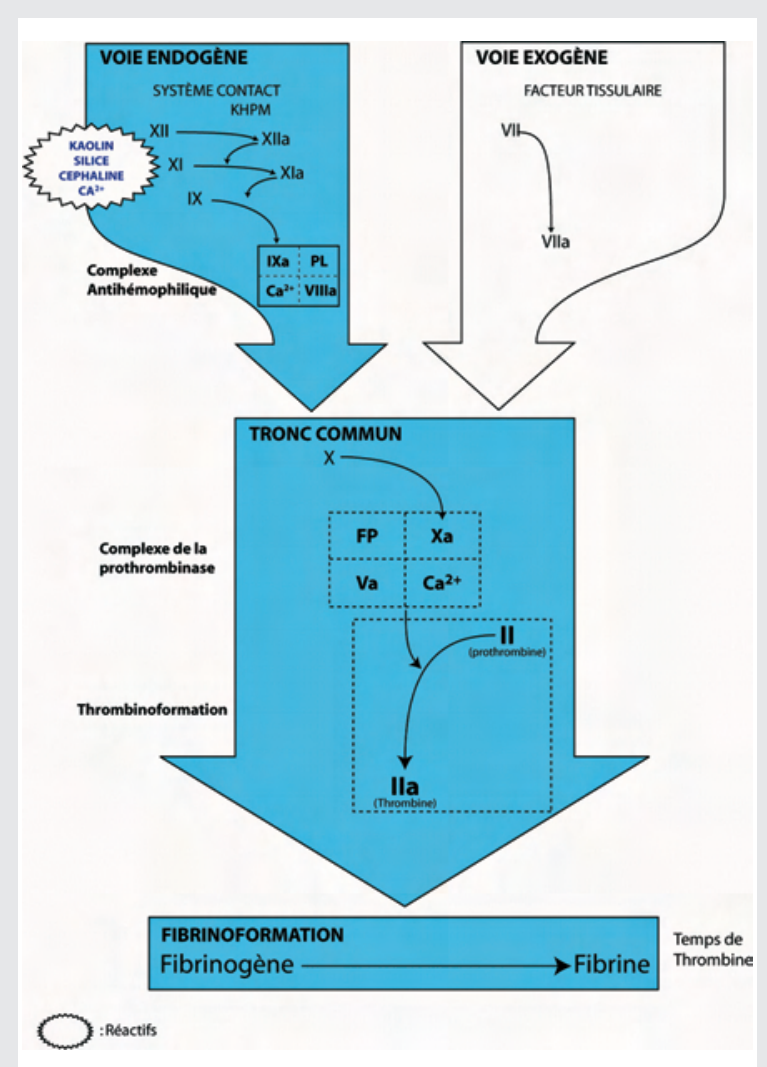

Figure 2 : Schéma du principe de réalisation d'un TCA : test global d'exploration de la voie endogène, du tronc commun et de la fibrinoformation. médecine

buccale

chirurgie

buccale

VOL. $9, N^{\circ} 3$

2003

page 187 
- permis la formation de complexes de la coagulation sur une surface phospholipidique par apport de céphaline ;

- rétablit la concentration physiologique en calcium ionisé (Ca2+).

Le TCA est exprimé en secondes. II est interprété par rapport à un temps de plasma témoin normal. Sa valeur normale ne doit pas dépasser plus de 6 à 8 secondes celle du témoin. Le rapport TCA du malade/TCA du témoin doit être privilégié par souci de standardisation des résultats. II doit être inférieur à 1,2.

\section{TEMPS DE QUICK (TQ) ET INTERNA- TIONAL NORMALIZED RATIO (INR)}

Le TQ est un test global d'exploration de la coagulation sanguine (Fig 3). II explore, in vitro :

- la voie exogène (ou voie du facteur tissulaire),

médecine

buccale

chirurgie

buccale

VOL. $9, \mathrm{~N}^{\circ} 3$ 2003

page 188
- le tronc commun (complexe de la prothrombinase, thrombinoformation, fibrinoformation).

Ce test consiste à mesurer le temps de coagulation, à $37^{\circ} \mathrm{C}$, d'un plasma pauvre en plaquettes, anticoagulé par recueil sur citrate de sodium $(0,113 \mathrm{M})$, après avoir :

- activé la voie du facteur tissulaire par un excès de thromboplastine ;

- rétablit la concentration physiologique en calcium ionisé (Ca2+).

Le TQ s'exprime en secondes. Il est interprété par rapport à un temps de plasma témoin normal. La normalité du TQ varie entre 10 et 14 secondes. Le TQ est le plus souvent exprimé en taux de prothrombine (TP) ou en INR pour le suivi biologique des patients traités par antagonistes de la vitamine $K(A V K)$.

Le TP exprime le TQ du patient par rapport à une droite étalon construite à partir de la mesure du TQ d'un plasma témoin normal à plusieurs dilutions. Le TP s'exprime en pourcentage. Par définition, le TQ du plasma témoin normal non dilué correspond à $100 \%$ d'activité prothrombinique. Celui obtenu sur le plasma dilué de moitié correspond à la valeur $50 \%$ et ainsi de suite. Le TP du patient correspond au taux de dilution du plasma témoin normal donnant le même temps. La valeur normale du TP varie entre 70 et $100 \%$. L'INR est le rapport du TQ du malade/TQ d'un

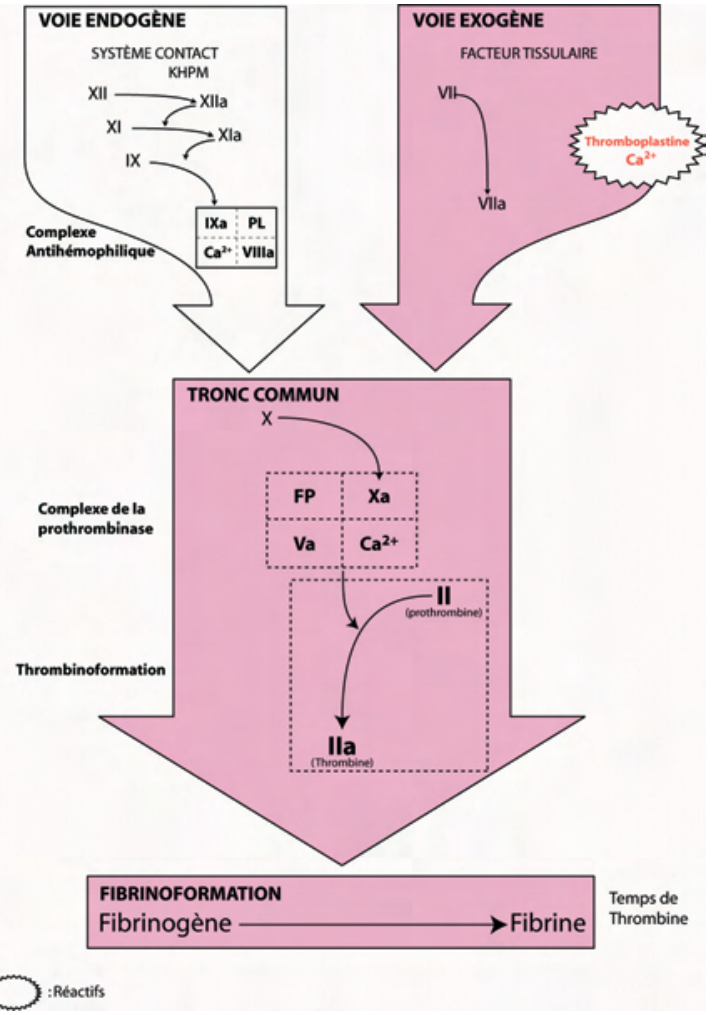

Figure 3 : Schéma du principe de réalisation d'un TP: test global d'exploration de la voie exogène, du tronc commun et de la fibrinoformation.

plasma témoin normal élevé à la puissance ISI (international sensitivity index). L'ISI est le coefficient de correction de la thromboplastine utilisée par le laboratoire par rapport à une thromboplastine internationale de référence. La valeur normale de l'INR est égale à 1. L'expression du TQ sous forme d'INR normalise les résultats entre laboratoires.

\section{ORIENTATIONS DIAGNOSTIQUES}

La démarche diagnostique devant une ou plusieurs anomalies des différents tests de l'hémostase (TS, TCA, TQ et INR) repose sur :

(1) une interprétation globale des résultats,

(2) une confrontation aux données cliniques (interrogatoire essentiellement).

\section{Allongement isolé du TS}

Cette situation est définie par un TS allongé, un TCA et un TQ normaux. II s'agit donc d'une anomalie limitée à l'hémostase primaire. L'orientation 
diagnostique repose alors sur une numération plaquettaire (NP). La valeur normale de la NP varie

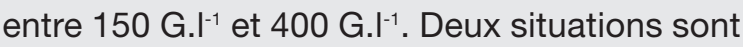
possibles :

\section{Numération plaquettaire diminuée}

Une NP inférieure à $150 \mathrm{G}^{-\mathrm{I}^{-1}}$ définit une thrombopénie.

- Pour une NP inférieure $50 \mathrm{G.l}^{-1}$, l'allongement du TS est constant et corrélé à la sévérité de la thrombopénie.

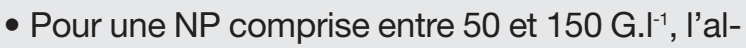
longement du TS ne peut s'expliquer par la seule thrombopénie. Une anomalie fonctionnelle des plaquettes (thrombopathie) doit alors être suspectée.

\section{Numération plaquettaire normale}

En l'absence d'une thrombopénie, un allongement du TS évoque une thrombopathie.

Les thrombopathies acquises sont les plus fréquentes, le plus souvent d'origine médicamenteuse (salicylés et autres inhibiteurs des fonctions plaquettaires). Les thrombopathies congénitales sont exceptionnelles et demandent des examens complémentaires spécifiques.

\section{Allongement isolé du TCA}

Cette situation est définie par une anomalie de la voie endogène de la coagulation sanguine. Par ailleurs, I'hémostase primaire est normale. Un allongement isolé du TCA peut s'expliquer par :

(1) la présence d'héparines non fractionnées (HNF) ou de bas poids moléculaires (HBPM) à posologie élevée ;

(2) un déficit isolé en un des facteurs de la voie endogène (XII, XI, IX et VIII) ;

(3) la présence d'anticorps anticoagulants circulants (AAC) de type antiphospholipidique.

L'hémophilie est la coagulopathie hémorragique grave la plus fréquente. II y a environ 5000 hémophiles en France. L'hémophilie est une anomalie constitutionnelle de la coagulation sanguine. L'anomalie est héréditaire par transmission récessive liée au chromosome $X$, les sujets masculins étant atteints et les féminins conducteurs. Elle est due à un déficit ou à une anomalie qualitative du facteur VIII (hémophilie A) ou du facteur IX (hémophilie B).

\section{Allongement isolé du TQ}

Cette situation est définie par une anomalie de la voie exogène de la coagulation sanguine avec une hémostase primaire normale. Un allongement isolé du TQ peut s'expliquer par :

(1) une insuffisance hépatocellulaire modérée ;

(2) un traitement par antagoniste de la vitamine $\mathrm{K}$ $(A V K)$ en début de traitement. En effet, le facteur VII est le facteur vitamine K-dépendant dont la demi-vie est la plus courte (5 à 7 heures). C'est donc celui dont le taux diminue en premier lors de l'instauration d'un traitement par AVK ;

(3) une hypovitaminose K pathologique (carence, malabsorption);

(4) exceptionnellement, un déficit isolé en facteur VII constitutionnel ou acquis (par exemple par anticorps anti-F.VII).

\section{Allongement combiné du TCA et du TQ}

Dans cette situation, la coagulation sanguine est altérée dans son ensemble et l'hémostase primaire demeure intacte (TS normal). Une exploration complémentaire par dosage du fibrinogène plasmatique est alors indispensable. Sa valeur

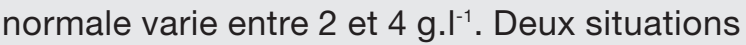
sont envisageables :

\section{Taux de fibrinogène diminué}

Lorsque le déficit en fibrinogène est modéré (entre 0,2 et $\left.1,5 \mathrm{g.l}^{-1}\right)$, le TS est normal. Un dosage spécifique des facteurs du complexe de la prothrombinase (II, V, X) est alors indiqué.

- Un déficit isolé en fibrinogène oriente vers une anomalie constitutionnelle (hypofibrinogènémie) ou vers une anomalie acquise (traitement par thrombolytiques).

- Un déficit du fibrinogène associé à d'autres déficits (II, $V$ et $X$ ) évoque soit une insuffisance hépatocellulaire modérée, soit une coagulation intravasculaire disséminée (CIVD).

- Lorsque le déficit en fibrinogène est sévère

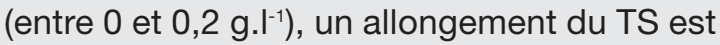
constant (voir allongement combiné du TS, TCA et $T Q$ ). \begin{tabular}{l}
$\begin{array}{l}\text { médecine } \\
\text { buccale } \\
\text { chirurgie } \\
\text { buccale }\end{array}$ \\
\hline VOL. $9, N^{\circ} 3$ \\
2003 \\
\hline page 189
\end{tabular} 


\section{Taux de fibrinogène normal}

Cette situation doit faire évoquer en premier lieu la prise d'antithrombotique (AVK ou HNF). Si l'interrogatoire est négatif, un dosage spécifique des facteurs de la prothrombinase (II, V, X) est indiqué :

- Une diminution isolée en facteur II, V ou X oriente vers un déficit constitutionnel ou acquis.

- Une diminution conjointe du II et du X (avec V normal) impose le dosage du facteur VII à la recherche d'une hypovitaminose $\mathrm{K}$ (carence, malabsorption intestinale, obstruction des voies biliaires).

\section{Allongement du TS associé à une anomalie de la coagulation sanguine}

\section{Allongement combiné du TS et du TCA}

médecine

buccale

chirurgie

buccale

VOL. $9, \mathrm{~N}^{\circ} 3$ 2003

page 190
Un allongement concomitant du TS et du TCA doit faire évoquer :

(1) une maladie de Willebrand,

(2) une thrombopénie consécutive à un traitement par héparines (HNF, HBPM). Une numération plaquettaire est indispensable pour évaluer l'importance de cet effet indésirable.

La maladie de Willebrand est la plus fréquente des anomalies constitutionnelles de l'hémostase (prévalence : 0,57 à 1,15\%). La transmission génétique est autosomale. Elle est liée à une anomalie qualitative et/ou quantitative du facteur Von Willebrand (vWF). Le vWF a deux fonctions essentielles : permettre les interactions des plaquettes avec la paroi vasculaire lésée, et assurer le transport et la stabilité de l'activité coagulante du facteur VIIIc dans le plasma.

\section{Allongement combiné du TS, du TCA et du TQ}

Cette situation est définie par une anomalie conjointe de l'hémostase primaire et de la coagulation sanguine. Elle oriente vers une perturbation globale de l'hémostase en rapport avec :

(1) une insuffisance hépatocellulaire sévère,

(2) une CIVD,

(3) exceptionnellement, un déficit majeur et isolé en fibrinogène (afibrinogénémie).

\section{CONCLUSION}

La facilité de la réalisation des examens biologiques de dépistage des anomalies de l'hémostase ne doit pas occulter le coût qu'ils représentent pour la collectivité. Leur recours doit donc se limiter à des indications réfléchies, résultant d'une évaluation préalable méthodique et précise.

En présence de valeurs anormales, les données issues de l'interrogatoire et/ou de l'examen clinique permettent le plus souvent d'orienter le diagnostic ou d'expliquer les variations constatées par un contexte pathologique ou thérapeutique connu. Dans le cas contraire, toute valeur anormale inexpliquée nécessite un bilan étiologique dans le cadre d'une prise en charge spécialisée. II ne faut pas oublier que ces troubles peuvent constituer les manifestations de processus pathologiques graves notamment malins.

\section{Bibliographie}

1 - GOUAULT-HEILMANN V. Aide -mémoire d'hémostase. éd : Médecine-Sciences Flammarion, Paris, 1999.

2 - LEROY J, POTRON G, SAMAMA M, GUILLIN MC, TOBELEM G, GRUEL Y. Hémostase et thrombose. $4^{e}$ édition. éd: la Simarre, Joué-lès-Tours, 1994.

3 - NAJMAN A, VERDY E, POTRON G, ISNARD F. Hématologie tome II. Éd: ellipses, Paris, 1994.

4 - SÉBAHOUN G. Hématologie clinique et biologique. Éd : Arnette Initiatives Santé, Vélizy-Villacoublay, 1998.

5 - Indications des examens préopératoires. Conférence d'experts. ANDEM, Paris, 1992.

6 - Evaluation de la prescription et de la réalisation des examens paracliniques préopératoires. ANAES, Paris, 1998. 